\title{
Optimization of Piezoresistive Motion Detection for Ambient NEMS Applications
}

\author{
Chaoyang Ti, ${ }^{1}$ Atakan Ari, ${ }^{1}$ Ezgi Orhan, ${ }^{1}$ Miguel Gonzalez, ${ }^{2}$ Cenk Yanik, ${ }^{3}$ Ismet I. Kaya, ${ }^{4}$ \\ M. Selim Hanay, ${ }^{5}$ IEEE, and Kamil L. Ekinci ${ }^{1, *}$ \\ ${ }^{1}$ Department of Mechanical Engineering, Division of Materials Science and Engineering, and the Photonics \\ Center, Boston University, Boston, Massachusetts, 02215, United States \\ ${ }^{2}$ Aramco Americas, Aramco Research Center-Houston, Houston, Texas, 77084, United States \\ ${ }^{3}$ SUNUM, Nanotechnology Research and Application Center, Sabanci University, Istanbul, 34956, Turkey \\ ${ }^{4}$ Faculty of Engineering and Natural Sciences, Sabanci University, Istanbul, 34956, Turkey \\ ${ }^{5}$ Department of Mechanical Engineering, and the National Nanotechnology Research Center (UNAM), \\ Bilkent University, Ankara, 06800, Turkey \\ Email: ${ }^{1}$ \{chaoyang, atakanba, ekinci* $\} @$ bu.edu, ${ }^{2}$ miguel.gonzalez@aramcoamericas.com, \\ 3,4 $\{$ cenkyanik, iikaya\}@ sabanciuniv.edu, 5 selimhanay@bilkent.edu.tr
}

\begin{abstract}
Electrical readout of nanomechanical motion in ambient pressure and temperature imposes an important challenge for emerging applications of nanoelectromechanical systems (NEMS). Here, we optimize a metallic piezoresistive motion transducer for NEMS resonators in air. The nanomechanical motion of the NEMS resonator serves as a signal down-mixer and enables the detection of the motional signal by a low-frequency circuit. A balanced circuit in the detection loop reduces some of the unwanted background and allows for detection without significant losses. We explore the detection parameter space and use an optimized parameter set to detect the fundamental, second and third harmonic resonances of a NEMS doubly-clamped beam resonator. Our simple circuit model agrees with experimental observations and points the way for further optimization.
\end{abstract}

Index Terms-NEMS, metallic piezoresistance, signal downmixing, motion transducer, background reduction

\section{INTRODUCTION}

Electrically detected nanoelectromechanical systems (NEMS) with integrated piezoresistive strain sensors are promising for a variety of applications [1] [2] [3]. So far, most such devices have been operated in vacuum [2] and some at low temperatures [4], because the high resonance quality $(Q)$ factors under these conditions allow for efficient detection. The reduction in $Q$ in ambient atmosphere [5] makes piezoresistive detection more susceptible to impedance mismatches [6] and parasitic effects [1], resulting in engineering challenges. However, many NEMS applications - such as environmental monitoring, bio-chemical threat detection and mechanical signal processing - are in ambient air. Here, we optimize piezoresistive detection for NEMS resonators with metallic piezoresistor transducers by implementing a down-mixing technique [7] in a balanced circuit. We show reduction in the background levels by optimizing the mix-down frequency in the balanced circuit.

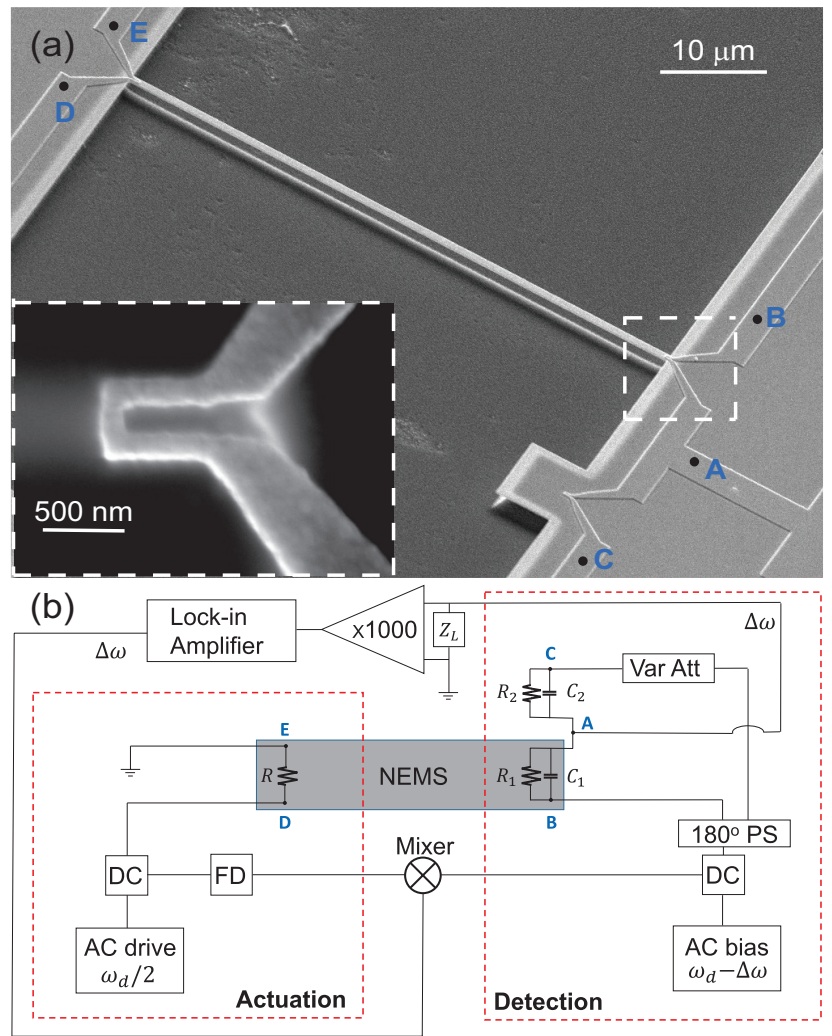

Fig. 1: (a) Scanning electron microscope image of the NEMS device used in this study, with a close-up (inset) image of the gold electrode for integrated piezoresistive detection and electrothermal actuation. (b) Schematic of the measurement circuit. The actuation and detection setups are shown in the left and right boxes, respectively. DC: directional coupler; PS: power splitter; FD: frequency doubler; Var Att: variable attenuator. $\mathrm{A}, \mathrm{B}, \mathrm{C}, \mathrm{D}$, and $\mathrm{E}$ ports on the circuit correspond to those on the device in (a). 


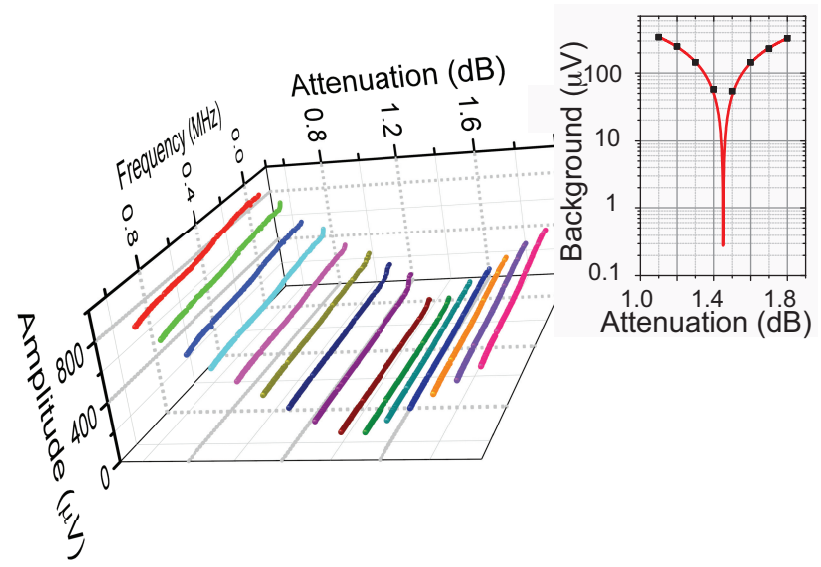

Fig. 2: Electrical response of the bridge without mechanical motion. The background is plotted as a function of relative attenuation between the arms of the bridge. The frequency is swept from $20 \mathrm{kHz}$ to $1 \mathrm{MHz}$ (bandwidth of the pre-amplifier). The inset shows the background levels (symbols) as a function of relative attenuation at $600 \mathrm{kHz}$. The fit (red curve) is based on Eq. (2) with the $R_{1}, R_{2}$ and $R_{L}$ values in text.

\section{RESUltS}

The doubly-clamped beam NEMS resonator used in this study with linear dimensions $l \times w \times t \approx 50 \mu \mathrm{m} \times 900 \mathrm{~nm} \times$ $100 \mathrm{~nm}$ is shown in Fig. 1. The NEMS device is patterned by electron-beam lithography on a 100-nm-thick high-stress silicon nitride on silicon wafer. Two u-shaped metal film resistors $(4 \mathrm{~nm} \mathrm{Cr} / 100 \mathrm{~nm} \mathrm{Au})$ with typical resistances of $\sim 20 \Omega$ are thermally deposited near the two ends of the NEMS for electrothermal actuation and piezoresistive detection (Fig. 1a, inset). The resistors are $600 \mathrm{~nm}$ long and $120 \mathrm{~nm}$ wide. The mechanical properties of the resonator are dominated by the tension in the silicon nitride layer [8].

To improve the signal-to-background ratio (SBR), we use the balanced detection circuit in Fig. 1b. The first nano-resistor $\left(R_{1}=17.6 \Omega\right)$ is the metallic piezoresistive transducer and is fabricated on top of the NEMS resonator between points A and $\mathrm{B}$; the second nano-resistor $\left(R_{2}=17.2 \Omega\right)$ with identical dimensions is for nulling purposes and is between points $\mathrm{A}$ and C. The parasitic capacitances $C_{1}$ and $C_{2}$ are on the order of tens of $\mathrm{pF}$. If $180^{\circ}$-out-of-phase voltages are applied to points $\mathrm{B}$ and $\mathrm{C}$, the voltage at point A will effectively be nulled [9]. Due to fabrication imperfections, the resistances $R_{1}$ and $R_{2}$ are slightly different. To further improve the SBR, we add a variable attenuator in the balanced circuit. The readout voltage at point $\mathrm{A}$ can be determined as

$$
V_{A}(\omega)=\left(-\frac{\left|V_{C}\right|}{Z_{1}}+\frac{\left|V_{B}\right|}{Z_{2}}\right) /\left(\frac{1}{Z_{L}}+\frac{1}{Z_{1}}+\frac{1}{Z_{2}}\right)
$$

where $V_{C}$ and $V_{B}$ are the voltages applied to $Z_{1}$ and $Z_{2}$, respectively, with $Z_{1}(\omega)=R_{1} /\left(1+j \omega R_{1} C_{1}\right)$ and $Z_{2}(\omega)=$ $R_{2} /\left(1+j \omega R_{2} C_{2}\right)$. The pre-amplifier has an input impedance $Z_{L}=100 \mathrm{M} \Omega \| 25 \mathrm{pF}$. When the operating frequency is in the MHz range, $\omega R_{1} C_{1} \ll 1$ and $\omega R_{2} C_{2} \ll 1$, resulting in

$$
V_{A} \approx\left(-\frac{\left|V_{C}\right|}{R_{1}}+\frac{\left|V_{B}\right|}{R_{2}}\right) /\left(\frac{1}{R_{L}}+\frac{1}{R_{1}}+\frac{1}{R_{2}}\right) .
$$

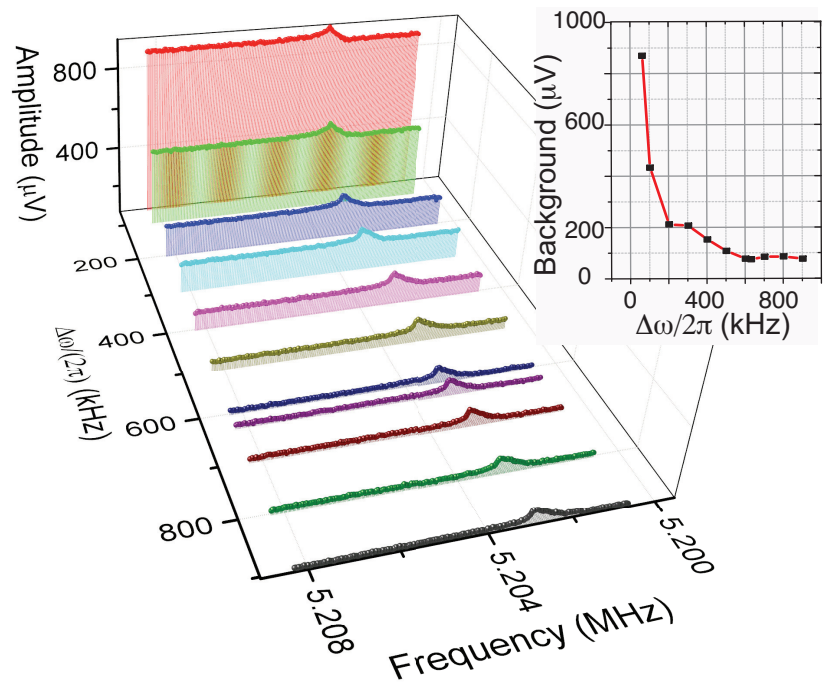

Fig. 3: Dependence of the measured signal on the intermediate frequency $\Delta \omega$. The same NEMS resonance is measured using different values of $\Delta \omega$. The inset shows the signal-tobackground ratio obtained by dividing the resonance amplitude on top of the background to the average background level.

We first optimize the detection circuit by tuning the variable attenuator in the balanced circuit in order to find the minimum background level. Fig. 2 shows the background level in the detection frequency band as a function of the relative attenuation between the two arms of the balanced circuit. The measured background voltages agree with the circuit model (inset) using the given values for the circuit elements.

Now, we turn to the electrothemal actuation of the NEMS device using the actuation circuit in Fig. 1b. When a current passes through the actuation metallic nano-resistor, the local heating induces bending of the beam toward the substrate due to the differential thermal expansion of gold and silicon nitride layers [7] [10]. When the temperature oscillates periodically at the frequency of a mechanical resonance at $\frac{\omega_{n}}{2 \pi}$ ( $n$ mode number) of the NEMS resonator, the resonance can be excited. The dissipated power, and hence the temperature, is $\propto I(t)^{2}$, where $I(t)$ is the AC current passing through the actuation loop. Thus, if the frequency of the actuation current is swept around $\frac{\omega_{n}}{4 \pi}$, the NEMS resonance at $\frac{\omega_{n}}{2 \pi}$ can be excited [7].

The vibration of the NEMS at $\frac{\omega_{n}}{2 \pi}$ causes a geometric deformation of the piezoresistor on the NEMS. The resistor undergoes a time-varying resistance change $\Delta R(t)$ at the frequency $\frac{\omega_{n}}{2 \pi}$ in response to the strain, with $R_{1} \gg \Delta R$. Because of parasitic capacitances [11], this piezoresistance signal $\Delta R(t)$ will be attenuated. To reduce this unwanted effect, we mix down the resonance signal to a lower intermediate frequency by using an AC biasing technique. Here, the NEMS is driven electromechanically at $\frac{\omega_{n}}{4 \pi}$ to vibrate at $\frac{\omega_{n}}{2 \pi}$, while an AC bias voltage at frequency $\frac{\omega_{n}-\Delta \omega}{2 \pi}$ is applied to point $C$ in Fig. 1b. Since $\Delta R(t) \propto e^{i \omega_{n} t}$ and the first-order current through the circuit is $I(t) \propto e^{i\left(\omega_{n}-\Delta \omega\right) t}$, the readout from the balanced circuit is down-mixed to $\frac{\Delta \omega}{2 \pi}$. The signal is then amplified by a pre-amplifier and detected by a lock-in amplifier (Fig. 1b). 


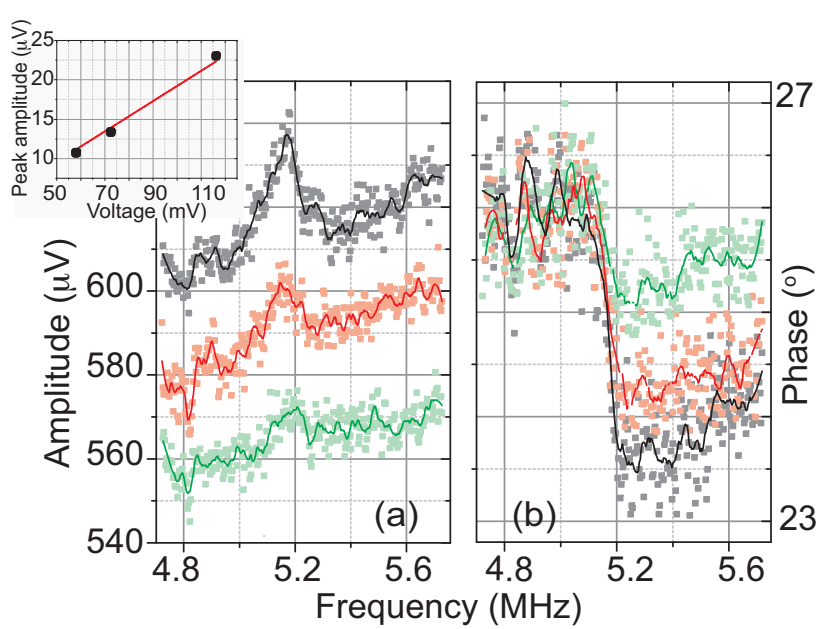

Fig. 4: (a) Amplitude of the NEMS fundamental resonance signal at $\sim 5.2 \mathrm{MHz}$ measured at different $\mathrm{AC}$ bias voltages and a fixed actuation voltage $(158 \mathrm{mV})$. The bias voltages are $58.2 \mathrm{mV}$ (green), $72.6 \mathrm{mV}$ (red), and $116.4 \mathrm{mV}$ (black). Inset shows linear dependence of peak amplitude on the bias voltage. (b) Corresponding phase shifts.

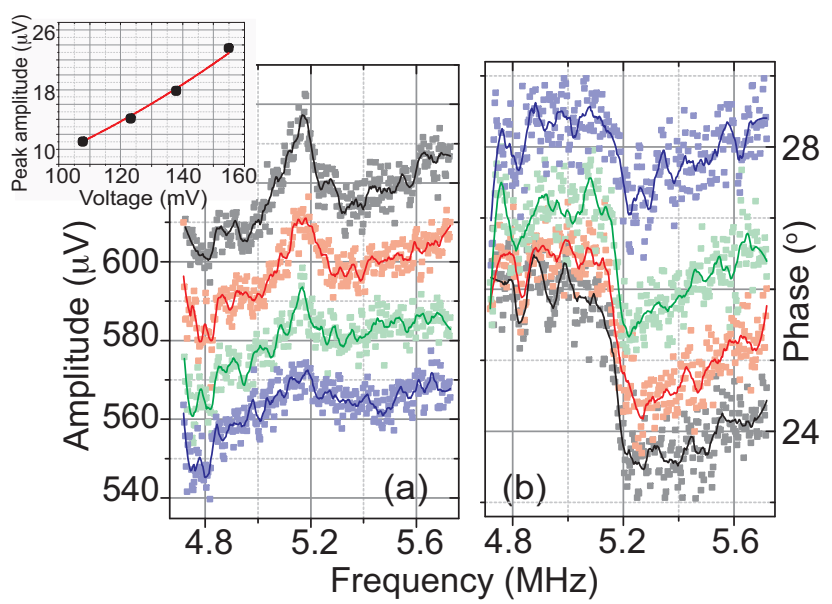

Fig. 5: (a) Amplitude of the NEMS fundamental resonance signal as a function of frequency at different actuation voltages with the same AC bias $(116.4 \mathrm{mV})$ applied to the detection port. The drive voltages are $107.8 \mathrm{mV}$ (blue), $123.3 \mathrm{mV}$ (green), $138 \mathrm{mV}$ (red), and $155 \mathrm{mV}$ (black squares). Inset shows the dependence of the peak amplitude on the drive voltage. (b) Corresponding phase shifts.

The background in our measurement setup depends on the value of the intermediate frequency $\frac{\Delta \omega}{2 \pi}$. We characterize this effect by changing $\frac{\Delta \omega}{2 \pi}$ while keeping every other parameter constant. Fig. 3 shows the background-to-signal ratio as a function of $\frac{\Delta \omega}{2 \pi}$ for the fundamental $(n=1)$ resonance at $\sim 5.2 \mathrm{MHz}$. The background first decreases as $\frac{\Delta \omega}{2 \pi}$ increases; it then saturates and increases again. Based on this, the minimum background is attained when $\frac{\Delta \omega}{2 \pi} \approx 630 \mathrm{kHz}$.

Figs. $4 \mathrm{a}$ and $4 \mathrm{~b}$ show the amplitude and phase of the fundamental $(n=1)$ resonance signal, respectively, as a function of frequency at three different $\mathrm{AC}$ bias voltages at a fixed

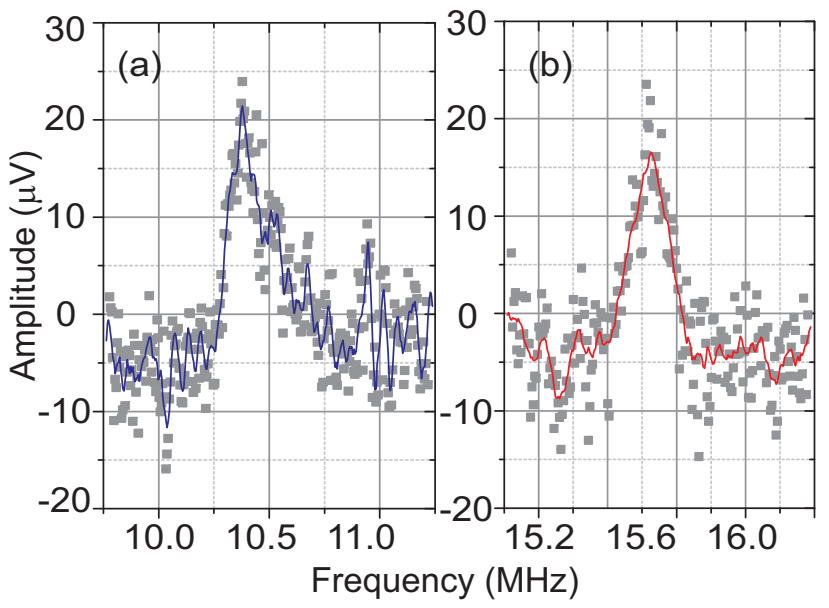

Fig. 6: Second (a) and third resonant modes (b) of the NEMS device at $10.4 \mathrm{MHz}$ and $15.5 \mathrm{MHz}$, respectively.

drive voltage in air. Increasing the bias voltage increases the current in the detection circuit and results in larger amplitudes. However, both the signal peak and the background increase. The inset shows the amplitude of the peak obtained after background subtraction. The peak amplitude shows a linear trend with the applied bias at a fixed motional amplitude level determined by the drive voltage. Conversely, Fig. 5 shows the resonance signal at different drive voltages at a fixed bias voltage. There is an increase of the background with the drive voltage, suggesting that there is cross-talk between the actuation and detection circuits. The inset shows the signal amplitude and suggests that the dependence on drive voltage is quadratic - as expected. Finally, high order modes can be measured in air after these optimizations. Figs. $6 \mathrm{a}$ and $6 \mathrm{~b}$ respectively show the $n=2$ and $n=3$ modes of the same NEMS resonator after background reduction. The measured frequencies at the second and third modes of the NEMS are 10.38 and $15.5 \mathrm{MHz}$, roughly twice and three times the fundamental resonance frequency.

\section{Conclusions}

Even though the down-mixing technique allows for detection at an intermediate frequency $\Delta \omega$ significantly different from the electromechanical excitation frequency $\frac{\omega_{n}}{2}$ and biasing frequency $\omega_{n}-\Delta \omega$, there still appears to be parasitic coupling between these signals. The balanced circuit in this work has allowed for reduction of some of the parasitic coupling. In addition, the selection of $\Delta \omega$ affects the background level in our circuit, providing optimal background around 600 $\mathrm{kHz}$. We expect these experimental results to be useful for further development of ambient NEMS devices.

\section{ACKNOWLEDGEMENTS}

This work was supported by the Aramco Services Company (A-0208-2019) and the US NSF (CBET 1604075 and CMMI 1934271). 


\section{REFERENCES}

[1] R. He, X. Feng, M. Roukes, and P. Yang, "Self-transducing silicon nanowire electromechanical systems at room temperature," Nano letters, vol. 8, no. 6, pp. 1756-1761, 2008.

[2] M. Li, H. X. Tang, and M. L. Roukes, "Ultra-sensitive nems-based cantilevers for sensing, scanned probe and very high-frequency applications," Nature nanotechnology, vol. 2, no. 2, p. 114, 2007.

[3] J. Jiwei, Z. Quanbin, Y. Heng, L. Tie, L. Xinxin, and W. Yuelin, "Doubly clamped nanobeam as a piezoresistive mass sensor," in SENSORS, 2007 IEEE. IEEE, 2007, pp. 1168-1171.

[4] M. S. Hanay, S. Kelber, A. Naik, D. Chi, S. Hentz, E. Bullard, E. Colinet, L. Duraffourg, and M. Roukes, "Single-protein nanomechanical mass spectrometry in real time," Nature nanotechnology, vol. 7, no. 9, p. 602, 2012.

[5] V. Kara, V. Yakhot, and K. L. Ekinci, "Generalized knudsen number for unsteady fluid flow," Physical review letters, vol. 118, no. 7, p. 074505 , 2017.

[6] I. Bargatin, E. Myers, J. Arlett, B. Gudlewski, and M. Roukes, "Sensitive detection of nanomechanical motion using piezoresistive signal downmixing," Applied Physics Letters, vol. 86, no. 13, p. 133109, 2005.

[7] I. Bargatin, I. Kozinsky, and M. Roukes, "Efficient electrothermal actuation of multiple modes of high-frequency nanoelectromechanical resonators," Applied Physics Letters, vol. 90, no. 9, p. 093116, 2007.

[8] A. T. Liem, A. B. Ari, J. G. McDaniel, and K. L. Ekinci, "An inverse method to predict nems beam properties from natural frequencies," Journal of Applied Mechanics, vol. 87, no. 6, 2020.

[9] K. Ekinci, Y. Yang, X. Huang, and M. Roukes, "Balanced electronic detection of displacement in nanoelectromechanical systems," Applied physics letters, vol. 81, no. 12, pp. 2253-2255, 2002.

[10] S. Timoshenko, "Analysis of bi-metal thermostats," Josa, vol. 11, no. 3 , pp. 233-255, 1925.

[11] T. Kouh, M. S. Hanay, and K. L. Ekinci, "Nanomechanical motion transducers for miniaturized mechanical systems," Micromachines, vol. 8, no. 4 , p. 108,2017 